Article

\title{
A Small U-Shaped Bending-Induced Interference Optical Fiber Sensor for the Measurement of Glucose Solutions
}

\author{
Yu-Lin Fang, Chen-Tung Wang and Chia-Chin Chiang * \\ Department of Mechanical Engineering, National Kaohsiung University of Applied Sciences, Kaohsiung 807, \\ Taiwan; fangiack@gmail.com (Y.-L.F.); 1101403104@gm.kuas.edu.tw (C.-T.W.) \\ * Correspondence: ccchiang@kuas.edu.tw; Tel.: +886-7-381-4526 (ext. 5340)
}

Academic Editors: Teen-Hang Meen, Shoou-Jinn Chang, Stephen D. Prior and Artde Donald Kin-Tak Lam Received: 30 July 2016; Accepted: 6 September 2016; Published: 9 September 2016

\begin{abstract}
The study proposes a small U-shaped bending-induced interference optical fiber sensor; this novel sensor is a probe-type sensor manufactured using a mechanical device, a heat source, optical fiber and a packaging module. This probe-type sensor overcomes the shortcomings of conventional optical fibers, including being difficult to repair and a tendency to be influenced by external forces. We manufactured three types of sensors with different curvature radiuses. Specifically, sensors with three radiuses $(1.5 \mathrm{~mm}, 2.0 \mathrm{~mm}$, and $3.0 \mathrm{~mm})$ were used to measure common water and glucose solutions with concentrations of between $6 \%$ and 30\% (the interval between concentrations was $4 \%$ ). The results show that the maximal sensitivity was $0.85 \mathrm{~dB} / \%$ and that the linearly-dependent coefficient was 0.925 . The results further show that not only can the small U-shaped bending-induced interference optical fiber sensor achieve high sensitivity in the measurement of glucose solutions, but that it can also achieve great stability and repeatability.
\end{abstract}

Keywords: whispering gallery mode; optical fiber sensor; glucose

\section{Introduction}

Optical fibers have often been utilized in communications [1] and data transmission [2]; however, optical fiber technology has advanced substantially in recent years, increasing its value and expanding its potential uses even further. Optical fiber has various advantages. For example, it is light, small in size, highly sensitive, highly flexible [3], highly resistant to electromagnetic interference (EMI) [4], and can be embedded into or attached to a wide range of structures [5]. Thus, optical fiber has strong potential for further development and application. In recent years, for example, various sensors manufactured from optical fiber have been used to not only measure various physical properties (such as temperature change [6], strain [7], vibration effects [8], etc.), but also concentration changes of various liquids [9].

Rayleigh [10] (an English physicist) proposed the whispering gallery mode (WGM) theory, and his research investigated, among other things, the transmission phenomena of acoustic waves along a curved wall. Subsequently, numerous scholars have investigated how to transmit acoustic waves in bent optical fiber according to Rayleigh's theory. In 2010, Wang et al. [11] proposed using a WGM refractive index sensor to measure the refractive indices of external media. In a related experiment, they used three optical fibers with different curvatures to measure the refractive indices of seven solutions with different concentrations at room temperature. In manufacturing each sensor, they removed the protective layer of the optical fiber and bent the optical fiber to form a ring with a diameter of $19 \mathrm{~mm}$, and then used glue to ensure that the shape of the optical fiber would be maintained. Next, the finished sensor was placed in the experimental solution for measurement. The experimental results showed 
that the refractive index sensitivity of the optical fiber sensor with a diameter of $19.3 \mathrm{~mm}$ could reach as high as $725.76 \mathrm{~nm} / \mathrm{RIU}$.

In 2012, Mathew et al. [12] proposed the use of bent optical fiber to manufacture a humidity sensor for use in a humidity measurement system. They packaged an optical fiber in a sensing layer and then placed the fiber on a movable platform in order to change the radius of the optical fiber through the movements of the platform. Next, they prepared several measurement environments with different humidity in order to observe the rate of energy change in the fiber; the advantages of the system included its low cost and quick rate of response, which made it very suitable as a humidity measurement sensor. In 2013, Nishimura and Tanabe [13] proposed the use of a spherical chamber made of optical fiber to measure the differences between tap water and pure water. More specifically, they changed the refractive index of the WGM and the surface of the chamber to attract nanoparticles in order to measure samples of tap water and pure water. The results showed that the device exhibited a wavelength shift of $89 \mathrm{pm}$ when measuring water, and the authors thus suggested that it had potential for use in monitoring water purity.

In 2013, Kwon and Steier [14] proposed a micro-ring-resonator-based glucose sensor consisting of two different micro-ring resonators. The authors measured both the temperature and concentration of glucose solutions simultaneously. In 2014, Bhardwaj et al. [15] proposed the dual taper-like fiber probe glucose sensor based on a Mach-Zehnder Interferometer (MZI) using the arc fusion splicer. Relatively high sensitivities are achieved by using the intensity interrogation methods. The sensitivity of the device is about $376.12 \mathrm{~nm} /$ RIU.

In 2014, Chao [16] proposed a U-shaped bending optical fiber glucose concentration sensor. The results showed that as the tested glucose concentration increased from $0 \%$ to $50 \%$, the spectrum shifted from short to long wavelengths. When the fiber diameter was $64 \mu \mathrm{m}$ and bend radius was $3.5 \mathrm{~mm}$, the optimal glucose concentration sensitivity was $0.520 \mathrm{~nm} / \%$.

The results of the studies reviewed above indicate that bending optical fiber sensors could measure changes in the refractive index of solutions. In fact, researchers have already found that sensors utilizing this design exhibit good sensitivity in solution measurements, including measurements of solution concentration and water quality. The present study expanded upon such research by using small U-shaped bending-induced interference optical fiber sensors of different sizes to test glucose solutions with different concentrations.

\section{Materials and Sensor Manufacturing Process}

As shown in Figure 1, we removed $60 \mathrm{~mm}$ of optical fiber protective layer from the middle section of a $300 \mathrm{~mm}$-long single mode fiber. Next, we fixed the two ends of the fiber to a movable platform consisting in part of a $300 \mathrm{~mm}$-long flexible aluminum board. More specifically, we fixed the ends of the fiber to a tension meter, and set the diameter of the optical fiber so that it would be $7 \mathrm{~mm}$ when the tension meter reached $12 \mathrm{~N}$. Next, we heated up the beat ends of the optical fiber. During the manufacturing process, the aluminum board bounced off because of the release of the potential energy caused by the tension, which in turn caused the optical fiber to pass through the holes and assume the diameter and the size that we had designed. Finally, the desired probe-type U-shaped fiber component was finished.

We used quartz glass to package the finished probe-type U-shaped fiber component, maintained the distance required to remove the optical fiber at the outlets of the capillary, and then used transparent super glue to fix the outlets of the tube in order to form an independent sensor; the optical fiber was fixed according to the design specifications. The advantage of this design was in the further fixation of the probe head, which allowed the influence of human-made and environmental factors to be reduced. At the same time, the optical fiber would not crack when used to measure a given liquid. 


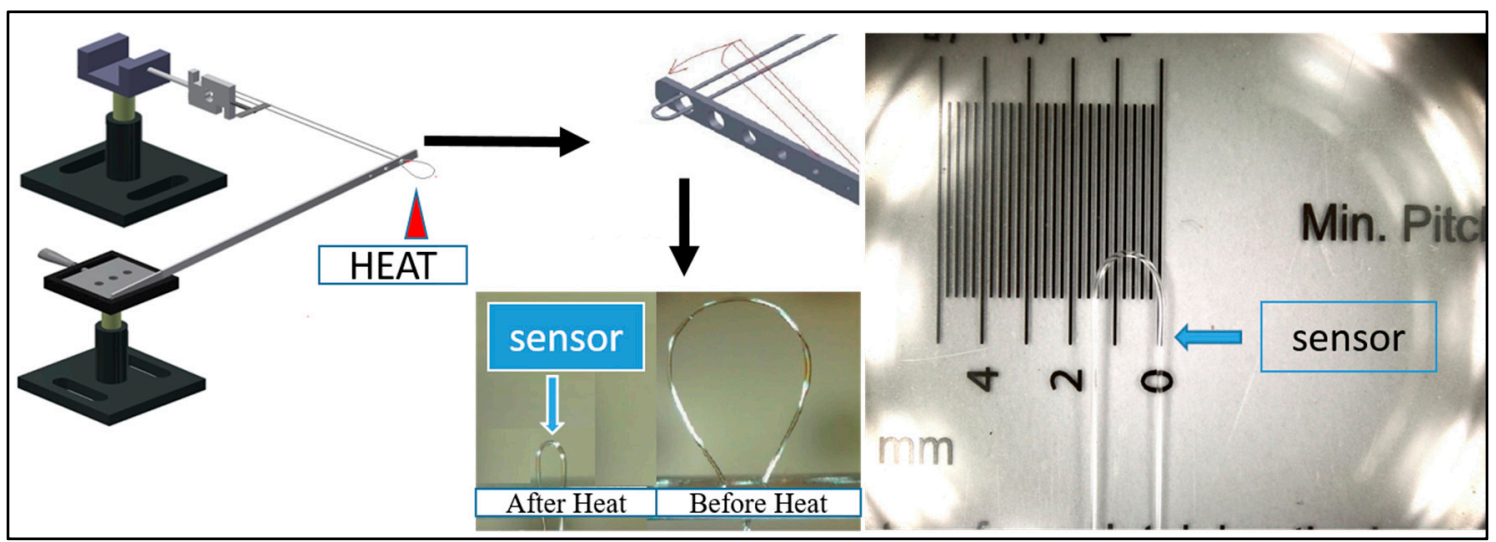

Figure 1. Schematic view of manufacturing process of small U-shaped bending-induced interference glucose concentration sensor.

\section{Experiment}

In the experiment in which the small U-shaped bending-induced interference optical fiber sensor was used to measure the refractive indices of glucose solutions, the sensor was first immersed into liquids with different refractive indices. The relationship between the given refractive index and the wavelength was then determined according to the phase change during reflection due to the refractive index difference between the casing of the optical fiber and the external liquid. Figure 2 shows the set-up of the equipment that we used with the small $U$-shaped bending-induced interference optical fiber sensor in the glucose solution concentration refractive index experiment. First, we affixed the glass tube to the height gage with double circular bars. Next, we affixed the small U-shaped bending-induced interference glucose concentration sensor to the glass tube fixer; one end of the sensor was connected to the optical spectrum analyzer (OSA), and the other end of the sensor was connected to the light source. Next, the OSA transferred the signal into the spectrum signal, and then the spectrum signal was shown on the PC monitor.

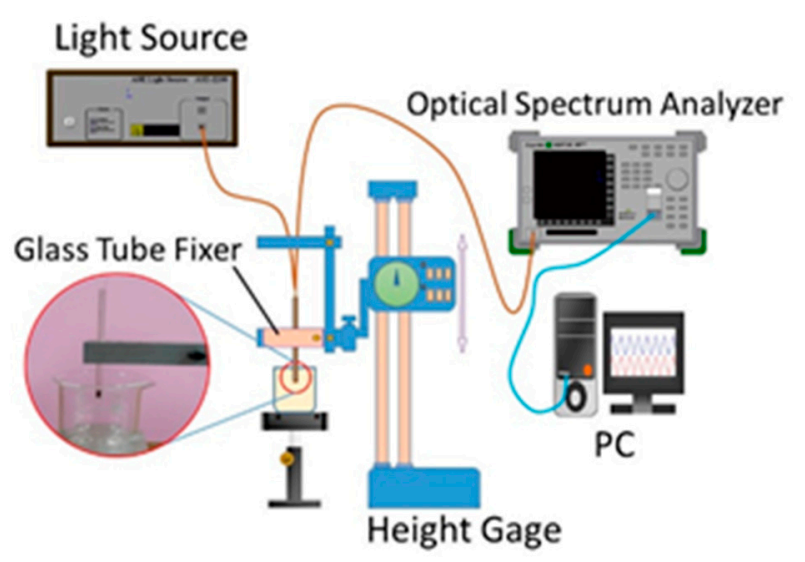

Figure 2. Experimental set-up for the small U-shaped bending-induced interference optical fiber sensor glucose solution concentration refractive index experiment.

Regarding the glucose solutions that were measured, we prepared several glucose solutions with concentrations ranging from $6 \%-30 \%$ (with intervals of $4 \%$ between solutions). Each solution was then placed in turn on the height-adjustable measurement table, and then the distance between the glass tube fixer of the height gage and the fluid level of the glucose solution in the beaker was adjusted as necessary until the sensor was immersed in the solution, allowing the refractive index measurement of that solution to be conducted. 


\section{Results and Discussion}

\subsection{Concentration Refractive Index Measurement of Solutions to be Measured}

The experimental process can be divided into two parts, including the glucose refractive index measurement by the Abbe refractometer and the water/glucose solution measurement by the small U-shaped bending-induced interference concentration sensor (the diameter and the bent radius of the optical fiber used were $125 \mu \mathrm{m}$ and $1.5 \mathrm{~mm}$, respectively).

First, we use the Abbe refractometer to measure the liquid refractive index of each glucose solution, and the measurement results indicated a linear relationship between the concentration and the refractive index, as shown in Figure 3. In addition, the sensitivity was $0.001 \mathrm{~dB} / \%$, and the linearly-dependent coefficient $\mathrm{R}^{2}$ was 0.999 , showing that the solutions used in the experiment can be recognized according to the solution accuracy.

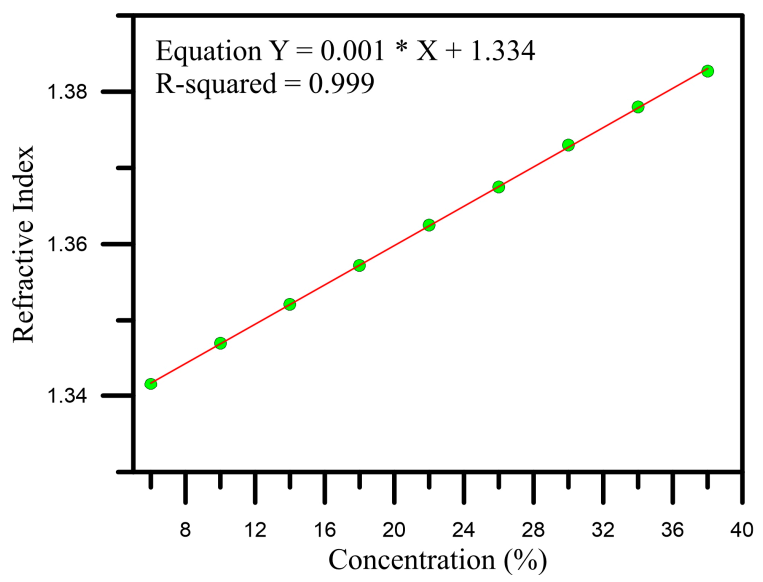

Figure 3. Diagram of the relationship between glucose solution concentration and refractive index.

\subsection{Concentration Test of Water/Glucose Solution}

We first immersed the sensor with a bent radius of $1.5 \mathrm{~mm}$ into water, and found that the central wavelength was $1491.75 \mathrm{~nm}$ and the transmission loss was $-33.075 \mathrm{~dB}$. Next, we measured glucose solutions with concentrations of $6 \%-30 \%$. When the concentration of the glucose solution was $6 \%$, the central wavelength was $1492.0 \mathrm{~nm}$. In contrast, when the concentration of the glucose solution was 30\%, the central wavelength was $1492.0 \mathrm{~nm}$, but the wavelength shift was $1.75 \mathrm{~nm}$. Thus, we could see that the long wavelength was shifted toward the direction of the short wavelength at the concentration wavelength position. In terms of the transmission loss, as the concentration was raised from $6 \%$ to $30 \%$, the transmission loss ranged from $-33.509 \mathrm{~dB}$ to $-35.827 \mathrm{~dB}$; thus, the energy loss tended to increase, as shown in Figure 4a.

When we used the sensor with a bent radius of 2.0 to measure the solutions, the wavelength also tended to be shifted toward the short wavelength as the concentration increased; specifically, the wavelength was shifted from $1531.25 \mathrm{~nm}(6 \%)$ to $1529.75 \mathrm{~nm}(30 \%)$. As such, the wavelength shift was $1.5 \mathrm{~nm}$, and the transmission loss grew from $35.395 \mathrm{~dB}$ to $36.687 \mathrm{~dB}$, as shown in Figure $4 \mathrm{~b}$.

When we used the sensor with bent radius of $3.0 \mathrm{~mm}$ to measure the solutions, the wavelength was shifted toward the long wavelength as the concentration was increased. Specifically, the wavelength was increased from $1521.5 \mathrm{~nm}(6 \%)$ to $1521.75 \mathrm{~nm}(30 \%)$. The wavelength shift was $0.25 \mathrm{~nm}$, and the transmission loss decreased from $-41.453 \mathrm{~dB}$ to $-41.275 \mathrm{~dB}$, as shown in Figure $4 \mathrm{c}$. However, when the concentration of the solution was $14 \%$, the wavelength was $1521.5 \mathrm{~nm}$ and the transmission loss was $-39.808 \mathrm{~dB}$.

When we used the sensor with a bent radius of $1.5 \mathrm{~mm}$ to measure the glucose solutions with concentrations between $6 \%$ and $30 \%$, the energy transmission loss was $-33.509 \mathrm{~dB}$. As the concentration 
was increased, a maximal energy loss of $-35.827 \mathrm{~dB}$ was found when the concentration of the glucose solution was $30 \%$, and the energy loss reached up to $2.291 \mathrm{~dB}$, as shown in Figure 5a. Furthermore, the sensitivity was $0.085 \mathrm{~dB} / \%$ and the linearity was 0.925 . The results for the sensor with a bent radius of $2.0 \mathrm{~mm}$ are shown in Figure 5b; the energy loss was $1.26 \mathrm{~dB}$, the sensitivity was $0.051 \mathrm{~dB} / \%$, and the linearity was 0.991 . For the sensor with a bent radius of $3.0 \mathrm{~mm}$, the transmission loss was $2.24 \mathrm{~dB}$, the sensitivity was $0.081 \mathrm{~dB} / \%$, and the linearity was 0.947 , as shown in Figure $5 \mathrm{c}$. According to the experimental results above, these sensors are very sensitive to changes in concentration.

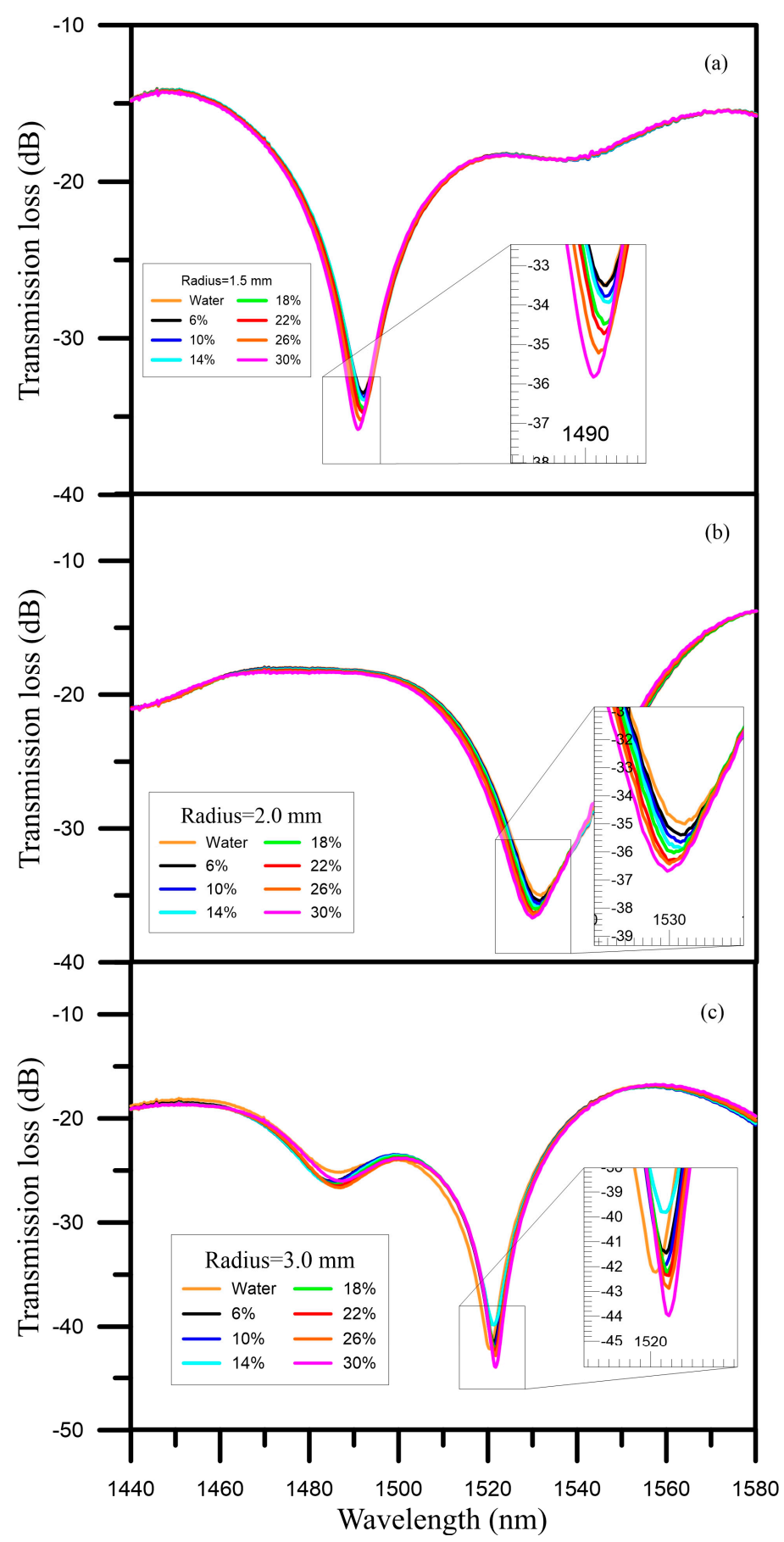

Figure 4. Concentration sensing spectrum diagram of glucose solution for (a) a bent radius $\mathrm{D}=1.5 \mathrm{~mm}$; (b) a bent radius $\mathrm{D}=2.0 \mathrm{~mm}$; and (c) a bent radius $\mathrm{D}=3.0 \mathrm{~mm}$. 


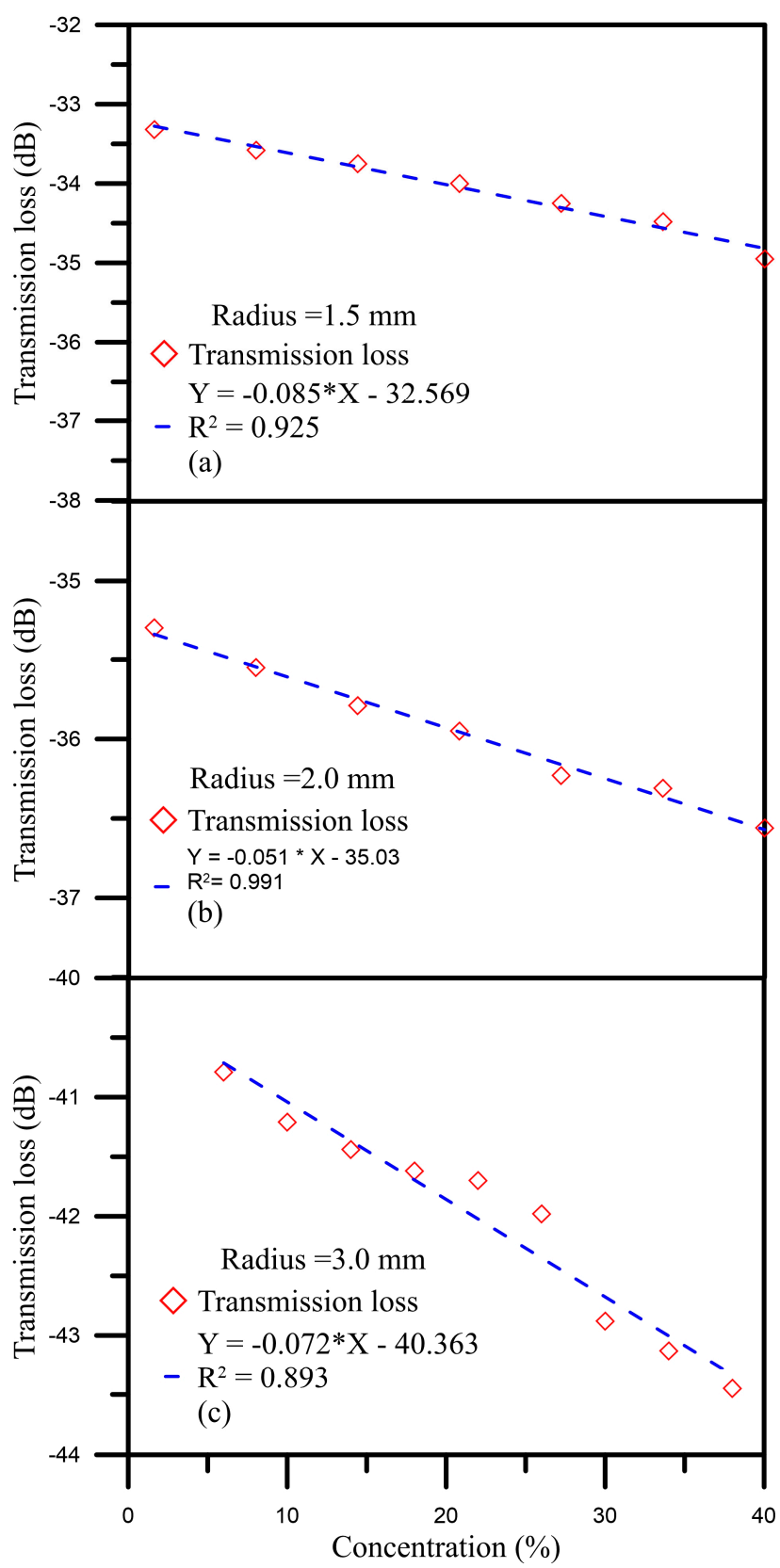

Figure 5. Diagram of the relationship between glucose solution concentration and transmission loss.

Next, we conducted repeatability and reproducibility experiments for the sensors. We used a sensor with a bent radius of $1.5 \mathrm{~mm}$ to measure glucose solutions with concentrations of $6 \%-30 \%$ for three cycles, and then performed a standard deviation analysis of the obtained data, the results of which are shown in Figure 6. This analysis found that the standard deviation of the transmission loss was within one standard deviation, as shown in Table 1. Accordingly, we can conclude that the sensors with radiuses of $1.5 \mathrm{~mm}, 2.0 \mathrm{~mm}$, and $3.0 \mathrm{~mm}$ have good reproducibility, and that these sensors can be repeatedly used for measurement. 


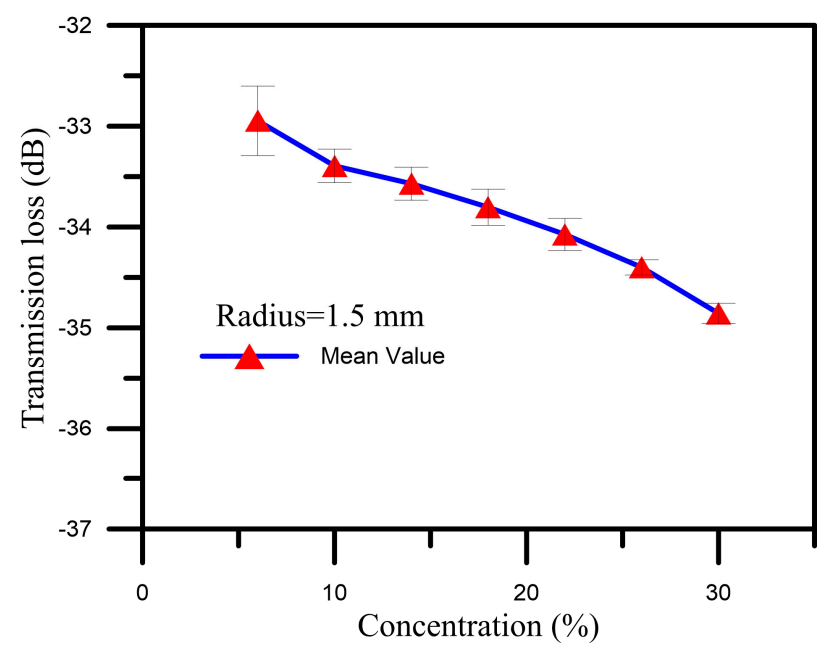

Figure 6. Diagram of the standard deviation analysis results for the obtained data.

Table 1. Wavelength and transmission loss standard deviation table for measurements made using the sensor with a radius of $1.5 \mathrm{~mm}$ to measure glucose solutions with concentrations of $6 \%-30 \%$ for three cycles.

\begin{tabular}{cccccccc}
\hline Concentration & $\mathbf{6 \%}$ & $\mathbf{1 0} \%$ & $\mathbf{1 4 \%}$ & $\mathbf{1 8 \%}$ & $\mathbf{2 2 \%}$ & $\mathbf{2 6 \%}$ & $\mathbf{3 0 \%}$ \\
\hline Transmission loss standard deviation $(\mathrm{dB})$ & 0.34 & 0.17 & 0.16 & 0.18 & 0.16 & 0.08 & 0.10 \\
\hline
\end{tabular}

\section{Conclusions}

This study utilized small U-shaped bending-induced interference optical fiber sensors. The respective bent radiuses of the sensors used were $1.5 \mathrm{~mm}, 2.0 \mathrm{~mm}$, and $3.0 \mathrm{~mm}$. Furthermore, the sensors were packaged in quartz glass tubes. We found that sensors manufactured in this way have greater storability, and that they do not suffer data distortion due to external forces; furthermore, such sensors can be manufactured in a short time and at a low cost.

In the experiments, we used glucose solutions to test the sensors. The measurement results showed that when the concentration of the glucose solutions was increased and when two sensors with bent radiuses of $1.5 \mathrm{~mm}$ were used to the measure the solution concentrations, the wavelength tended to be shifted from a long wavelength to a short wavelength. In terms of transmission loss, the result showed that the concentration wavelength and the transmission loss of the sensor with a bent radius of $1.5 \mathrm{~mm}$ was predictable. Moreover, the transmission loss standard deviation results showed that the accuracy of the sensor was high.

The study found that when used to measure glucose solution concentrations, the small U-shaped bending-induced interference optical fiber sensor with $\mathrm{D}=1.5 \mathrm{~mm}$ achieved the best performance in terms of transmission loss and measurement accuracy. The above experiments further showed that the small U-shaped bending-induced interference sensor constitutes a highly-sensitive and convenient glucose concentration sensor. In addition, the sensor also exhibited good repeatability and stability. In summary, this type of sensor is an excellent sensor and can be further developed for use as a real-time concentration monitoring sensor.

Acknowledgments: This work was supported by the National Science Council, Taiwan (grant number MOST 105-2623-E-151-002-D and 105-2218-E-212 -001).

Author Contributions: Yu-Lin Fang designed the experiments and analyzed the data. Chen-Tung Wang conducted the experiments. Chia-Chin Chiang also conducted the experiments, in addition to analyzing the experimental data and writing the paper.

Conflicts of Interest: The authors declare no conflict of interest. 


\section{References}

1. Amiri, I.S.; Nikoukar, A.; Ali, J. Nonlinear chaotic signals generation and transmission within an optical fiber communication link. IOSR J. Appl. Phys. 2013, 3, 52-57. [CrossRef]

2. Zhang, J.; Yu, J.; Chi, N.; Dong, Z.; Li, X.; Chang, G.K. Multichannel 120-Gb/s data transmission over $2 \times 2$ MIMO fiber-wireless link at W-band. IEEE Photon. Technol. Lett. 2013, 25, 780-783. [CrossRef]

3. Urick, V.J.; Diehl, J.F.; Singley, J.M.; Sunderman, C.E.; Williams, K.J. Long-reach analog photonics for military applications. Opt Photonics News. 2014, 25, 36-43. [CrossRef]

4. Gong, H.; Yang, X.; Ni, K.; Zhao, C.L.; Dong, X. An optical fiber curvature sensor based on two peanut-shape structures modal interferometer. IEEE Photonics Technol. Lett. 2014, 26, 22-24. [CrossRef]

5. Holmes, C.; Gates, J.C.; Smith, P.G. Planarised optical fiber composite using flame hydrolysis deposition demonstrating an integrated FBG anemometer. Opt. Express 2014, 22, 32150-32157. [CrossRef] [PubMed]

6. Mihailov, S.J. Fiber Bragg grating sensors for harsh environments. Sensors 2012, 12, 1898-1918. [CrossRef] [PubMed]

7. Long, F.; Zhu, A.; Shi, H. Recent advances in optical biosensors for environmental monitoring and early warning. Sensors 2013, 1310, 13928-13948. [CrossRef] [PubMed]

8. Morshed, A.H.E.; El-Sayed, I.M. Monitoring of vibrations using multimode optical fiber sensors. In Proceedings of the 2016 33rd National Radio Science Conference (NRSC), Aswan City, Egypt, 22-25 February 2016; pp. 384-389.

9. Zhang, W.; Gong, Y.; Ge, J.; Hua, B.; Zhang, H.; Ye, L. An optical fiber point liquid level sensor. In Proceedings of the AOPC 2015: Optical Fiber Sensors and Applications, Beijing, China, 5-7 May 2015.

10. Rayleigh, L. CXII. The problem of the whispering gallery. Philos. Mag. 1910, 20, 1001-1004. [CrossRef]

11. Wang, P.; Semenova, Y.; Li, Y.; Wu, Q.; Farrell, G. A macrobending single mode fiber refractive index sensor for low refractive index liquids. Photonics Lett. Pol. 2010, 2, 67-69.

12. Mathew, J.; Semenova, Y.; Farrell, G. Relative Humidity Sensor Based on an Agarose-Infiltrated Photonic Crystal Fiber Interferometer. IEEE J. Sel. Top. Quantum Electron. 2012, 18, 1553-1559. [CrossRef]

13. Nishimura, J.; Takasumi, T. Study on detection of contamination of pure water using silica microsphere. In Proceedings of the Lasers and Electro-Optics Europe, 2013 Conference on and International Quantum Electronics Conference, Munich, Germany, 12-16 May 2013.

14. Kwon, M.S.; Steier, W.H. Glucose sensor based on two distinct microring resonators. In Proceedings of the Australian Conference on Optical Fibre Technology (ACOFT) and Opto-Electronics Communications Conference (OECCC), Darling Harbour, Sydney, Australia, 7-10 July 2008.

15. Bhardwaj, V.; Singh, V.K. Fabrication and characterization of cascaded tapered Mach-Zehnder interferometer for refractive index sensing. Sens. Actuators A Phys. 2016, 244, 30-34. [CrossRef]

16. Chao, J.C. Whispering Gallery Mode Based Optical Fiber Sensor for Measuring Concentration of Glucose. Master's Thesis, National Kaohsiung University of Applied Sciences, Kaohsiung, Taiwan, 2014.

(C) 2016 by the authors; licensee MDPI, Basel, Switzerland. This article is an open access article distributed under the terms and conditions of the Creative Commons Attribution (CC-BY) license (http:/ / creativecommons.org/licenses/by/4.0/). 